\title{
REVIEW
}

Open Access

\section{Footwear and insole design features that reduce neuropathic plantar forefoot ulcer risk in people with diabetes: a systematic literature review}

Sayed Ahmed(D, Alex Barwick, Paul Butterworth and Susan Nancarrow

\begin{abstract}
Background: In people with diabetes, offloading high-risk foot regions by optimising footwear, or insoles, may prevent ulceration. This systematic review aimed to summarise and evaluate the evidence for footwear and insole features that reduce pathological plantar pressures and the occurrence of diabetic neuropathy ulceration at the plantar forefoot in people with diabetic neuropathy.

Methods: Six electronic databases (Medline, Cinahl, Amed, Proquest, Scopus, Academic Search Premier) were searched in July 2019. The search period was from 1987 to July 2019. Articles, in English, using footwear or insoles as interventions in patients with diabetic neuropathy were reviewed. Any study design was eligible for inclusion except systematic literature reviews and case reports. Search terms were diabetic foot, physiopathology, foot deformities, neuropath*, footwear, orthoses, shoe, footwear prescription, insole, sock*, ulcer prevention, offloading, foot ulcer, plantar pressure.

Results: Twenty-five studies were reviewed. The included articles used repeated measure $(n=12)$, case-control $(n=$ 3), prospective cohort $(n=2)$, randomised crossover $(n=1)$, and randomised controlled trial $(\operatorname{RCT})(n=7)$ designs. This involved a total of 2063 participants. Eleven studies investigated footwear, and 14 studies investigated insoles as an intervention. Six studies investigated ulcer recurrence; no study investigated the first occurrence of ulceration. The most commonly examined outcome measures were peak plantar pressure, pressure-time integral and total contact area. Methodological quality varied. Strong evidence existed for rocker soles to reduce peak plantar pressure. Moderate evidence existed for custom insoles to offload forefoot plantar pressure. There was weak evidence that insole contact area influenced plantar pressure.

Conclusion: Rocker soles, custom-made insoles with metatarsal additions and a high degree of contact between the insole and foot reduce plantar pressures in a manner that may reduce ulcer occurrence. Most studies rely on reduction in plantar pressure measures as an outcome, rather than the occurrence of ulceration. There is limited evidence to inform footwear and insole interventions and prescription in this population. Further high-quality studies in this field are required.
\end{abstract}

Keywords: Diabetic foot, Footwear, Insoles, Plantar pressure

\footnotetext{
* Correspondence: s.ahmed.13@student.scu.edu.au; sahmed50@gmail.com School of Health and Human Sciences, Southern Cross University, Billinga, Queensland 4225, Australia
} 


\section{Background}

Foot ulcers are a common consequence of diabetes due to the development of peripheral neuropathy, peripheral vascular disease, limited joint mobility and foot deformity [1-6]. Nearly $34 \%$ of persons with diabetes will develop a foot ulcer in their lifetime [7]. This can lead to infection and amputation; diabetes is the main reason for non-traumatic lower limb amputation [8, 9]. Previous foot ulcer or amputation is a risk of future amputation [1, 3, 5, 10]. Additional risk factors include higher Body Mass Index (BMI), and structural foot deformities $[2-4,6]$, such as hammertoes and hallux valgus $[11,12]$.

Diabetic peripheral neuropathy (DPN) is the central risk factor for the development of plantar foot ulceration [13]. Over $30 \%$ of persons with diabetes will develop DPN [14], the incidence increasing with age $[15,16]$. DPN can affect the autonomic, sensory and motor nervous systems. Sensory neuropathy interrupts the protective feedback mechanism of touch and pain [17]. Motor neuropathy results in compromised muscle innervation, reduction in strength, and combined with limited joint mobility, the development of foot deformities. These deformities may lead to an increase in plantar foot pressures, particularly in the forefoot [18-21]. Autonomic neuropathy leads to diminished sweating and changes to skin perfusion, leading to dry skin and hyperkeratosis. As skin integrity is compromised, patients are more susceptible to trauma which may precipitate a diabetic foot ulcer [21-24].

Neuropathic ulcers in diabetic feet occur mostly at the plantar forefoot $[11,25,26]$ and correspond to areas of peak plantar pressure [27]. Bennetts et al. [28] demonstrated that most peak pressure areas are located in the forefoot regions in this population. Limited range of motion at the forefoot joints is also likely to contribute to the peak plantar pressures (PPP) observed in this region [29]. For this reason, plantar pressure mapping is used to guide footwear and insole manufacture and judge their effectiveness [30].

Reducing plantar pressures is considered a key factor for wound healing and prevention of ulcer recurrence $[31,32]$. Footwear and insoles are an essential treatment modality for offloading these pressures [33, 34]. The desired offloading threshold should be $>30 \%$ reduction in dynamic in-shoe plantar pressure from the baseline or $<200 \mathrm{kPa}$ to ensure ulcer-free survival at the forefoot [35]. This systematic review aimed to summarise and evaluate the evidence for footwear and insole features that reduce pathological plantar pressures and the occurrence of diabetic neuropathy ulceration at the plantar forefoot in people with diabetic neuropathy.

\section{Methods}

The systematic search was performed according to the Preferred Reporting Items for Systematic Reviews and Meta-Analysis (PRISMA) Statement [36].

\section{Search strategy}

In July 2019, six electronic databases were searched (Medline, Cinahl, Amed, Proquest, Scopus, Academic Search Premier) using medical subject headings followed by a keyword subject heading. The search period was from 1987 to July 2019. The search terms can be seen in Fig. 1 and Supplementary file 1.

\section{Eligibility criteria}

All studies included in the systematic review were obtained from full-text peer-reviewed journals published in English. Studies that did not use footwear or insole as a mode of intervention for long term offloading were excluded. Letters to the editor, opinion pieces, conference proceedings, and editorials were also excluded. All study designs except systematic reviews and case reports were eligible for inclusion. The titles and abstracts of the articles were screened by one reviewer (SA). Full-text articles were reviewed based on the following criteria: i, participants were adult (> 18 years), had diabetes; ii, all or some of the participants had neuropathy and foot deformity, history of plantar forefoot ulcers but no Charcot foot, history of heel ulcer or active foot ulcers; iii, studies used footwear or insoles as a long-term offloading intervention; iv, the outcome of the study was either (re)occurrence of forefoot ulcer or change in forefoot plantar pressure outcomes; $v$, the footwear or insole interventions had to be sufficiently described to be able to draw useful conclusions; vi, conventional materials and manufacturing techniques were used; and vii, closed-in footwear was used. The reference lists of studies obtained through the database search were also searched to identify relevant citations.

\section{Quality assessment}

Quality assessment was performed independently by two reviewers ( $\mathrm{SA}$ and $\mathrm{AB}$ ). The quality assessment form was adapted from the McMaster Critical Review Form Quantitative Studies [37].

\section{Results}

The literature search identified 1787 articles. Twentyfive articles met the eligibility criteria to be included in the review (Fig. 2). The study designs included repeated measures $(n=12)$, case-control $(n=3)$, prospective cohort $(n=2)$, randomised crossover $(\mathrm{n}=1)$, and RCT $(n=7)$ studies.

Study characteristics are shown in Tables 1 and 2 . 


\begin{tabular}{llll}
\hline Or & Explode & Explode & Explode \\
Diabetic Foot & Footwear/orthoses & Ulcer prevention \\
$\qquad \begin{array}{lll}\text { physiopathology } \\
\text { foot deformities }\end{array}$ & shoe & offloading \\
neuropath* & footwear prescription & foot ulcer \\
& sock* & plantar pressure \\
And & &
\end{tabular}

Fig. 1 Search terms used to select the studies

\section{Participants and settings}

The participants were over 18 years of age, and the sample sizes ranged from 10 to 299. All participants in treatment groups had diabetes, and the majority had neuropathy. Participants had active or healed plantar foot ulcers, amputation, foot deformities, increased barefoot plantar pressure, or peripheral vascular disease. Most (88\%) of the studies recruited participants from developed countries within high-risk foot clinics and 12\% from developing countries [63]. Study duration ranged from a single session to 5 years.

\section{Intervention}

Eleven studies [30, 38, 39, 51, 52, 56-58, 60, 61] used footwear and insoles as the intervention. Of these, three studies [38, 57, 61] used footwear which was manufactured according to a consensus-based algorithm proposed by Dahmen et al. [53]. One study [52] specifically examined footwear rocker sole profiles. High footwear upper design feature was investigated by one study [51], and it reported that higher upper increased contact area but did not improve pressure reduction at the forefoot area.

Fourteen studies [30, 38-40, 42, 44, 46, 48, 49, 51, $53,58,61,62]$ reported on the prescribers, manufacturers and modifiers of the therapeutic footwear and insoles. The footwear prescribers reported in the studies were rehabilitation physicians $[30,38]$, diabetologist, podologist [61], podiatric physician [49]. The manufacturers for therapeutic footwear were

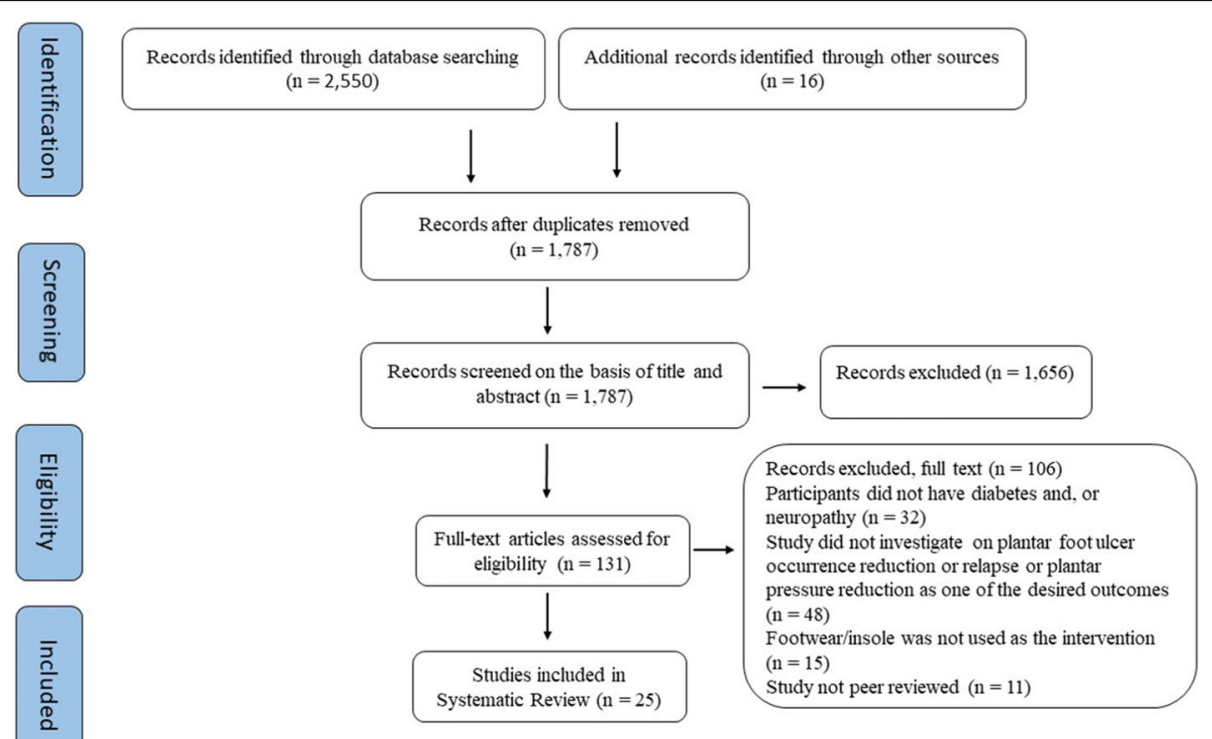

Fig. 2 PRISMA Study Selection Flow Diagram 
Table 1 Characteristics of the selected studies that used pressure reduction as the primary outcome measure

\begin{tabular}{|c|c|c|c|c|c|c|c|c|}
\hline Author, date & Location & Study design & $\begin{array}{l}\text { Follow up } \\
\text { period }\end{array}$ & $\begin{array}{l}\text { Sample } \\
\text { size }\end{array}$ & $\begin{array}{l}\text { Sample } \\
\text { characteristics }\end{array}$ & $\begin{array}{l}\text { Intervention \& } \\
\text { Comparison }\end{array}$ & $\begin{array}{l}\text { Outcome } \\
\text { measures }\end{array}$ & Result \\
\hline $\begin{array}{l}\text { Arts et al. } \\
2012 \text { [38] }\end{array}$ & Netherlands & $\begin{array}{l}\text { Repeated } \\
\text { measures }\end{array}$ & $\begin{array}{l}\text { Same } \\
\text { day }\end{array}$ & $\begin{array}{l}171 \\
(336 \mathrm{ft})\end{array}$ & $\begin{array}{l}\text { Diabetic } \\
\text { neuropathy } \\
\text { Previous } \\
\text { plantar ulcer }\end{array}$ & $\begin{array}{l}\text { Custom-made } \\
\text { footwear } \\
\text { Semi-customised } \\
\text { footwear } \\
\text { Barefoot }\end{array}$ & $\begin{array}{l}\text { Peak plantar } \\
\text { pressure (PPP) } \\
\text { of }<200 \mathrm{kPa} \\
\text { considered } \\
\text { successful }\end{array}$ & $\begin{array}{l}\text { Custom-made footwear } \\
\text { is least effective in } \\
\text { pressure reduction } \\
(<200 \mathrm{KPa} \text { ) at forefoot } \\
\text { compared to midfoot } \\
\text { and known ulcer } \\
\text { locations ( } 29 \% \text { vs } \\
81 \text { and } 62 \%)\end{array}$ \\
\hline $\begin{array}{l}\text { Arts et al. } \\
2015 \text { [39] }\end{array}$ & Netherlands & $\begin{array}{l}\text { Repeated } \\
\text { measures }\end{array}$ & $\begin{array}{l}\text { Same } \\
\text { day }\end{array}$ & 85 & $\begin{array}{l}\text { Diabetic } \\
\text { neuropathy } \\
\text { Previous } \\
\text { plantar foot } \\
\text { ulcer }\end{array}$ & $\begin{array}{l}\text { Various footwear } \\
\text { modifications to } \\
\text { custom or } \\
\text { semi-custom } \\
\text { footwear } \\
\text { Footwear before } \\
\text { modification }\end{array}$ & $\begin{array}{l}\% \text { plantar } \\
\text { pressure } \\
\text { reduction }\end{array}$ & $\begin{array}{l}\text { MP, local cushion and } \\
\text { plastazote top cover } \\
\text { reduce PP respectively } \\
\text { by } 15.9,15,14.2 \% \text { and } \\
\text { combinedly } 24 \text { and } \\
22 \% \text { at the forefoot. }\end{array}$ \\
\hline $\begin{array}{l}\text { Bus et al. } \\
2011[30]\end{array}$ & Netherlands & $\begin{array}{l}\text { Repeated } \\
\text { measures }\end{array}$ & $\begin{array}{l}\text { Not } \\
\text { reported }\end{array}$ & 23 & $\begin{array}{l}\text { Diabetic } \\
\text { Neuropathy, } \\
\text { Foot } \\
\text { deformity } \\
\text { Foot ulcer }\end{array}$ & $\begin{array}{l}\text { Fully custom-made } \\
\text { footwear and insoles }\end{array}$ & $\begin{array}{l}\text { In-shoe plantar } \\
\text { pressure reduction } \\
\text { by more than 25\% } \\
\text { (Criteria A) or } \\
\text { below the } \\
\text { absolute value of } \\
200 \mathrm{kPa} \text { (Criteria B) }\end{array}$ & $\begin{array}{l}\mathrm{MB} \text { or } \mathrm{MP} \text {, replacing } \\
\text { the top cover, early } \\
\text { rocker can reduce } \\
\text { pressure at hallux and } \\
\text { metatarsal area ranging } \\
\text { from } 10.1 \text { to } 18.6 \% \text { as an } \\
\text { individual modification. }\end{array}$ \\
\hline $\begin{array}{l}\text { Bus et al. } \\
2004 \text { [40] }\end{array}$ & Netherlands & $\begin{array}{l}\text { Repeated } \\
\text { measure }\end{array}$ & $\begin{array}{l}\text { Not } \\
\text { reported }\end{array}$ & 20 & $\begin{array}{l}\text { Diabetic } \\
\text { Neuropathy, } \\
\text { History of } \\
\text { healed } \\
\text { plantar foot } \\
\text { ulcers } \\
\text { Foot } \\
\text { deformity }\end{array}$ & $\begin{array}{l}\text { Insoles; } 9.5 \mathrm{~mm} \text { thick } \\
\text { flat PPT insole and } \\
\text { custom-made insoles } \\
\text { out of open-cell } \\
\text { urethane foams of } \\
\text { hardness } 60-80 \text {. } \\
\text { Custom-made insoles } \\
\text { were made by } \\
\text { CADCAM process. }\end{array}$ & $\begin{array}{l}\text { Plantar pressure } \\
\text { reduction } \\
\text { FTI }\end{array}$ & $\begin{array}{l}\text { Custom-made insoles } \\
\text { reduce plantar pressure } \\
\text { and FTI significantly at } \\
\text { medial and lateral heal, } \\
\text { MTH1 and FTI at lateral } \\
\text { MTHs when compared } \\
\text { with flat PPT insoles. }\end{array}$ \\
\hline $\begin{array}{l}\text { Charanya } \\
\text { et al. } \\
2004 \text { [41] }\end{array}$ & India & $\begin{array}{l}\text { Case-control } \\
\text { study }\end{array}$ & $\begin{array}{l}6 \\
\text { months }\end{array}$ & 25 & $\begin{array}{l}\text { Diabetic } \\
\text { Neuropathy } \\
\text { History of } \\
\text { active and } \\
\text { healed } \\
\text { plantar ulcers } \\
\text { Non-diabetic } \\
\text { (Control) }\end{array}$ & $\begin{array}{l}\text { Footwear with an } \\
\text { insole } \\
\text { made of } 12 \mathrm{~mm} \mathrm{MCR} \text {, } \\
\text { shore value } 20^{\circ} \text {, } \\
\text { Toughened rocker } \\
\text { profile rubber outsole }\end{array}$ & $\begin{array}{l}\text { Foot sole } \\
\text { hardness reduced } \\
\text { close to normal, } \\
\text { shore value } 20^{\circ}\end{array}$ & $\begin{array}{l}\text { Plantar ulcers healed } \\
\text { in three-four weeks, } \\
\text { foot sole skin hardness } \\
\text { reduced to } 25-30 \text { from } \\
45 \text { to } 50 \text { shore values. }\end{array}$ \\
\hline $\begin{array}{l}\text { Guldemond } \\
\text { et al. 2007 } \\
{[42,43]}\end{array}$ & Netherlands & $\begin{array}{l}\text { Repeated } \\
\text { measures }\end{array}$ & $\begin{array}{l}\text { Not } \\
\text { reported }\end{array}$ & 17 & $\begin{array}{l}\text { Diabetic } \\
\text { Neuropathy } \\
\text { Higher } \\
\text { barefoot } \\
\text { plantar } \\
\text { pressure } \\
(\geq 700 \mathrm{kPa})\end{array}$ & $\begin{array}{l}\text { Insole with various } \\
\text { height arch supports } \\
\text { and with and without } \\
\text { a metatarsal dome }\end{array}$ & $\begin{array}{l}\text { In-shoe plantar } \\
\text { pressure reduction } \\
(36 \% \text { \& 39\%), } \\
\text { Walking } \\
\text { convenience on a } \\
\text { 10-point rating } \\
\text { scale }\end{array}$ & $\begin{array}{l}\text { Extra arch support and } \\
\text { MD are respectively } \\
\text { effective in } 39 \% \text { \& } 36 \% \\
\text { pressure reduction in } \\
\text { central and medial } \\
\text { regions of the forefoot }\end{array}$ \\
\hline $\begin{array}{l}\text { Hastings et al. } \\
2007[44]\end{array}$ & USA & $\begin{array}{l}\text { Repeated } \\
\text { measure }\end{array}$ & $\begin{array}{l}22 \\
\text { months }\end{array}$ & 20 & $\begin{array}{l}\text { Diabetic } \\
\text { Neuropathy } \\
\text { History of } \\
\text { plantar foot } \\
\text { ulcers } \\
\text { No active } \\
\text { foot ulcers } \\
\text { No Charcot } \\
\text { neuropathy }\end{array}$ & $\begin{array}{l}\text { Three footwear } \\
\text { conditions; extra depth } \\
\text { footwear with 1) Total } \\
\text { Contact Insoles (TCI), 2) } \\
\text { TCI with proximal } \\
\text { Metatarsal Pad } \\
\text { (MP), 3) TCI with } \\
\text { distal MP, } \\
\text { CT Scan }\end{array}$ & $\begin{array}{l}\text { PPP } \\
\text { CT Scan for } \\
\text { positioning of MP } \\
\text { against MTHs }\end{array}$ & $\begin{array}{l}\text { Highest (57\%) PPP } \\
\text { reduction occurred at } \\
\text { 2nd MTH when MP } \\
\text { placed at } 10.6 \mathrm{~mm} \\
\text { proximal to MTH line. } \\
\text { Variable PPP under the } \\
\text { 2nd MTH varied } \\
\text { between } 32 \pm 16 \% \\
\text { when positioning of } \\
\text { MP varies between } \\
6.1 \text { mm to } 10.6 \mathrm{~mm} \\
\text { proximal to MTH line. }\end{array}$ \\
\hline $\begin{array}{l}\text { Lin et al. } \\
2013[45]\end{array}$ & China & $\begin{array}{l}\text { Repeated } \\
\text { measure }\end{array}$ & $\begin{array}{l}\text { Not } \\
\text { reported }\end{array}$ & 26 & $\begin{array}{l}\text { Diabetic } \\
\text { Neuropathy }\end{array}$ & $\begin{array}{l}\text { Insole with pre-plug } \\
\text { removal, post-plug } \\
\text { removal, and post-plug } \\
\text { removal + arch support }\end{array}$ & $\begin{array}{l}\text { Mean peak } \\
\text { pressure (MPP), } \\
\text { maximum force, } \\
\text { contact area }\end{array}$ & $\begin{array}{l}\text { Removing insole plug } \\
\text { is effective in offloading } \\
\text { MPP by } 32.3 \% \text { and } \\
\text { adding arch support } \\
\text { reduces further } 9.5 \% \text { at } \\
\text { the forefoot }\end{array}$ \\
\hline $\begin{array}{l}\text { Lott et al. } \\
2006 \text { [46] }\end{array}$ & USA & $\begin{array}{l}\text { Repeated } \\
\text { measure }\end{array}$ & $\begin{array}{l}\text { Not } \\
\text { reported }\end{array}$ & 20 & $\begin{array}{l}\text { Diabetic } \\
\text { Neuropathy } \\
\text { History of } \\
\text { midfoot or } \\
\text { forefoot } \\
\text { plantar ulcers }\end{array}$ & $\begin{array}{l}\text { Four different } \\
\text { conditions; 1) } \\
\text { Barefoot, } \\
\text { 2) Footwear, 3) } \\
\text { Footwear + TCl, 4) } \\
\text { Footwear + TCl + MP }\end{array}$ & $\begin{array}{l}\text { Plantar pressure } \\
\text { reduction } \\
\text { Soft tissue } \\
\text { thickness (STT) }\end{array}$ & $\begin{array}{l}\text { PP \& ST strain under } \\
\text { 2nd MTH are highest at } \\
\text { the barefoot condition } \\
\text { and lowest at footwear + } \\
\text { TCl + MP condition. } \\
\text { Mean PP for all four } \\
\text { conditions under 2nd }\end{array}$ \\
\hline
\end{tabular}


Table 1 Characteristics of the selected studies that used pressure reduction as the primary outcome measure (Continued)

\begin{tabular}{|c|c|c|c|c|c|c|c|c|}
\hline Author, date & Location & Study design & $\begin{array}{l}\text { Follow up } \\
\text { period }\end{array}$ & $\begin{array}{l}\text { Sample } \\
\text { size }\end{array}$ & $\begin{array}{l}\text { Sample } \\
\text { characteristics }\end{array}$ & $\begin{array}{l}\text { Intervention \& } \\
\text { Comparison }\end{array}$ & $\begin{array}{l}\text { Outcome } \\
\text { measures }\end{array}$ & Result \\
\hline & & & & & & & & $\begin{array}{l}\text { MTH is } 272 \mathrm{kPa} \text {, } \\
173 \mathrm{kPa}, 140 \mathrm{kPa} \\
\text { and } 98 \mathrm{kPa} \text {. }\end{array}$ \\
\hline $\begin{array}{l}\text { Martinez-Santos } \\
\text { et al. } 2019 \text { [47] }\end{array}$ & UK & $\begin{array}{l}\text { Repeated } \\
\text { measure }\end{array}$ & $\begin{array}{l}\text { Not } \\
\text { reported }\end{array}$ & 60 & $\begin{array}{l}\text { Diabetic } \\
\text { Neuropathy } \\
\text { No previous } \\
\text { ulcers }\end{array}$ & $\begin{array}{l}\text { Insole with three } \\
\text { different metatarsal bar } \\
\text { (MB) positioning, two } \\
\text { different types } \\
\text { of materials }\end{array}$ & PPP & $\begin{array}{l}\text { Maximum pressure } \\
\text { reduction can be } \\
\text { achieved by positioning } \\
\text { metatarsal bar at } 72 \% \\
\text { length of insole, } \\
\text { irrespective of material } \\
\text { type }\end{array}$ \\
\hline $\begin{array}{l}\text { Mueller et al. } \\
2006 \text { [48] }\end{array}$ & USA & $\begin{array}{l}\text { Repeated } \\
\text { measure }\end{array}$ & $\begin{array}{l}\text { Not } \\
\text { reported }\end{array}$ & 20 & $\begin{array}{l}\text { Diabetic } \\
\text { Neuropathy } \\
\text { history of } \\
\text { plantar ulcers }\end{array}$ & $\begin{array}{l}\text { Three footwear } \\
\text { conditions: 1) Footwear, } \\
\text { 2) Footwear with TCl, } \\
\text { and 3) Footwear } \\
\text { with TCI + MP }\end{array}$ & $\begin{array}{l}\text { PPP } \\
\text { PTI } \\
\text { STT }\end{array}$ & $\begin{array}{l}\mathrm{TCl} \text { and metatarsal pad } \\
\text { caused reductions of } \\
\text { pressure under the } \\
\text { metatarsal heads }\end{array}$ \\
\hline $\begin{array}{l}\text { Owings et al. } \\
2008 \text { [49] }\end{array}$ & USA & $\begin{array}{l}\text { Repeated } \\
\text { measure }\end{array}$ & $\begin{array}{l}\text { Not } \\
\text { reported }\end{array}$ & 20 & $\begin{array}{l}\text { Diabetic } \\
\text { Neuropathy } \\
\text { Higher } \\
(>750 \mathrm{kPa}) \\
\text { barefoot } \\
\text { plantar } \\
\text { pressure at } \\
\text { MTH region }\end{array}$ & $\begin{array}{l}\text { Three different type } \\
\text { custom-made insoles } \\
\text { (X, Y from shape-based } \\
\text { and Z combined foot } \\
\text { shape with plantar } \\
\text { pressure data). } \\
\text { Footwear with rigid } \\
\text { rocker sole and } \\
\text { flexible sole }\end{array}$ & $\begin{array}{l}\text { Peak pressure } \\
\text { FTI }\end{array}$ & $\begin{array}{l}\text { Shape and } \\
\text { pressure-based insoles } \\
\text { (Z) showed improved } \\
\text { offloading by } 32 \text { and } \\
21 \% \text {, PTI reduction } 40 \\
\text { and } 34 \% \text { when } \\
\text { compared to } \\
\text { shape-only-based } \\
\text { insoles } \\
\text { (X-Polypropylene base, } \\
\text { Y- EVA base). A similar } \\
\text { trend was observed in } \\
\text { flexible and rocker } \\
\text { bottom shoes for the } \\
\text { same insoles. }\end{array}$ \\
\hline $\begin{array}{l}\text { Paton et al. } \\
2012 \text { [50] }\end{array}$ & UK & RCT & 18 months & 119 & $\begin{array}{l}\text { Neuropathic } \\
\text { diabetic foot } \\
\text { ulceration }\end{array}$ & $\begin{array}{l}\text { Prefabricated and } \\
\text { custom-made insole }\end{array}$ & $\begin{array}{l}\text { In-shoe pressure } \\
\text { reduction, PTI, } \\
\text { forefoot rate of } \\
\text { load, total contact } \\
\text { area }\end{array}$ & $\begin{array}{l}\text { Prefab versus custom } \\
\text { insoles, } \mathrm{PPP} \geq 6 \%\end{array}$ \\
\hline $\begin{array}{l}\text { Praet et al. } \\
2003 \text { [51] }\end{array}$ & Netherlands & $\begin{array}{l}\text { Repeated } \\
\text { measure }\end{array}$ & $\begin{array}{l}\text { Not } \\
\text { reported }\end{array}$ & 10 & $\begin{array}{l}\text { Diabetic } \\
\text { Neuropathy } \\
\text { No active } \\
\text { ulcer, No } \\
\text { major foot } \\
\text { deformities }\end{array}$ & $\begin{array}{l}\text { Three different types } \\
\text { of footwear designs }\end{array}$ & $\begin{array}{l}\text { Peak pressure } \\
\text { reduction at } \\
\text { multiple areas } \\
\text { under the foot }\end{array}$ & $\begin{array}{l}\text { Rocker sole can offload } \\
\text { the forefoot area } \\
\text { by } 65 \%\end{array}$ \\
\hline $\begin{array}{l}\text { Preece et al. } \\
2017 \text { [52] }\end{array}$ & UK & Case-control & $\begin{array}{l}\text { Not } \\
\text { reported }\end{array}$ & 168 & $\begin{array}{l}\text { Diabetic } \\
\text { Neuropathy } \\
(n=17) \\
\text { Healthy } \\
\text { control } \\
(N=66)\end{array}$ & $\begin{array}{l}\text { Eight types of rocker } \\
\text { sole design }\end{array}$ & $\begin{array}{l}\text { Pressure reduction } \\
\text { threshold of } \\
\leq 200 \mathrm{kPa}\end{array}$ & $\begin{array}{l}\text { Rocker apex position at } \\
52 \%, 20^{\circ} \text { rocker angle, } \\
95^{\circ} \text { apex angle yields } \\
\text { effective offloading } \\
\text { at most }\end{array}$ \\
\hline $\begin{array}{l}\text { Tang et al. } \\
2014 \text { [53] }\end{array}$ & Sweden & RCT & $\begin{array}{l}\text { Two } \\
\text { years }\end{array}$ & 114 & $\begin{array}{l}\text { Diabetic } \\
\text { neuropathy } \\
\text { Angiopathy } \\
\text { Foot } \\
\text { deformities } \\
\text { Previous } \\
\text { ulcers or } \\
\text { amputation }\end{array}$ & $\begin{array}{l}\text { Three types of insoles, } \\
\text { custom made ( } 35 \& 55^{\circ} \\
\text { shore hardness EVA) vs } \\
\text { prefab insoles with } \\
\text { hardcore EVA + soft } \\
\text { microfiber top cover } \\
\text { (Control) }\end{array}$ & $\begin{array}{l}\text { PPP } \\
\text { PTI }\end{array}$ & $\begin{array}{l}\text { The overall PPP for the } \\
\text { insoles was between } \\
180 \mathrm{kPa} \text { to } 211 \mathrm{kPa} \text {, PTI } \\
\text { differences } 14 \mathrm{kPa} / \mathrm{sec} \& \\
20 \mathrm{kPa} / \mathrm{sec} \text { with Control. }\end{array}$ \\
\hline $\begin{array}{l}\text { Teffler et al. } \\
2017 \text { [54] }\end{array}$ & UK & $\begin{array}{l}\text { Randomised } \\
\text { crossover }\end{array}$ & $\begin{array}{l}\text { Not } \\
\text { reported }\end{array}$ & 20 & $\begin{array}{l}\text { Diabetic } \\
\text { neuropathy } \\
\text { Increased } \\
\text { forefoot } \\
\text { plantar } \\
\text { pressure } \\
\text { No Charcot } \\
\text { foot or partial } \\
\text { amputation }\end{array}$ & $\begin{array}{l}\text { Three types of insoles } \\
\text { 1) Standard } \\
\text { (Shape-based), milled } \\
\text { insoles, } \\
\text { 2) Milled, virtually } \\
\text { optimised insoles and } \\
\text { 3) 3D printed virtually } \\
\text { optimised insoles }\end{array}$ & PPP & $\begin{array}{l}\text { Virtually optimised } \\
\text { insole reduced PPP by } \\
\text { a mean of } 41.3 \mathrm{kPa} \text { for } \\
\text { milled and } 40.5 \mathrm{kPa} \text { for } \\
3 \mathrm{D} \text { printed insoles in } \\
\text { the same participants' } \\
\text { group. }\end{array}$ \\
\hline
\end{tabular}


Table 1 Characteristics of the selected studies that used pressure reduction as the primary outcome measure (Continued)

\begin{tabular}{|c|c|c|c|c|c|c|c|c|}
\hline Author, date & Location & Study design & $\begin{array}{l}\text { Follow up } \\
\text { period }\end{array}$ & $\begin{array}{l}\text { Sample } \\
\text { size }\end{array}$ & $\begin{array}{l}\text { Sample } \\
\text { characteristics }\end{array}$ & $\begin{array}{l}\text { Intervention \& } \\
\text { Comparison }\end{array}$ & $\begin{array}{l}\text { Outcome } \\
\text { measures }\end{array}$ & Result \\
\hline $\begin{array}{l}\text { Tsung et al. } \\
2004 \text { [55] }\end{array}$ & China & Case-control & $\begin{array}{l}\text { Not } \\
\text { reported }\end{array}$ & 14 & $\begin{array}{l}\text { Diabetic } \\
\text { neuropathy } \\
\text { No Charcot } \\
\text { foot or partial } \\
\text { amputation } \\
\text { Control: no } \\
\text { foot } \\
\text { deformity }\end{array}$ & $\begin{array}{l}\text { Five support conditions } \\
\text { including } \\
\text { footwear-only, flat } \\
\text { insoles; and three } \\
\text { custom-made insoles } \\
\text { with three } \\
\text { weight-bearing } \\
\text { conditions; 1) Full } \\
\text { weight-bearing (FWB), } \\
\text { 2) Semi-weight-bearing } \\
\text { (SWB) and } \\
\text { 3) Non-weight-bearing } \\
\text { (NWB) }\end{array}$ & $\begin{array}{l}\text { MPP } \\
\text { PTI } \\
\text { Mean contact } \\
\text { area }\end{array}$ & $\begin{array}{l}\text { For 2-3 MTH regions, } \\
\text { SWB insoles yield } \\
\text { maximum offloading } \\
\text { comparing to two } \\
\text { other insoles type. For } \\
\text { MTH1, NWB insoles } \\
\text { provide maximum } \\
\text { offloading. FWB insoles } \\
\text { show maximum PTI } \\
\text { comparing to NWB \& } \\
\text { SWB conditions. NWB } \\
\text { insoles provide } \\
\text { maximum arch support } \\
\text { and contoured shaped insoles }\end{array}$ \\
\hline
\end{tabular}

orthopaedic shoe technicians $[30,38,39,51,61]$, and orthopaedic shoemakers $[40,42,58]$, where orthopaedic shoe technicians have similar training like certified pedorthists [30]. Reported insole manufacturers or modifiers were orthotic technician [53], pedorthist [44, 49], pedorthist or orthotist $[46,48,49,62]$.

Fourteen studies [40, 42, 44, 46-50, 53-55, 59, 62, 64] used insoles as a primary intervention in standardised or participant's footwear. All studies reported on the type of footwear they used with varying descriptions of the design features and almost all studies reported on the description of insole design features used by the studies respectively, except Preece et al. [52]. Studies that are focused on the insole as a primary intervention has used prefabricated extra-depth footwear or regular retail footwear [40, 42, 44-50, 53-55, 62].

Insole features have been described by some studies [39, $41,45,47,49,50,53,54,56,59,60,62,64$ ] such as base, mid-layer, and top cover materials. The same authors also assessed hardness, thickness, casting and manufacturing technique, metatarsal dome or metatarsal bar, and arch support. Ten studies [40-42, 47, 48, 53, 55, 56, 59, 64] examined insole material thickness and hardness. Other components of insole configurations reported were application of metatarsal pad, metatarsal dome, or metatarsal bar [30, $39,40,42,44,46-48,53,57,61]$ and their positioning [42, $44,46-48,53]$, arch support [30, 39, 40, 42, 51, 53, 55, 57, 61], top cover [30, 39, 42, 49-51, 53-57, 59, 61, 62, 64], adding local cushion to insole $[39,49,57,61,62]$. The size of the metatarsal dome or pad used by the studies is between 5 to $11 \mathrm{~mm}[42,44,47]$ in height, 66 to $74 \mathrm{~mm}$ in length, and 51 to $63 \mathrm{~mm}$ width [44]. The positioning of the metatarsal dome, bar or pad was between 5 to $10.6 \mathrm{~mm}$ proximal to MTHs $[42,44,46]$ and at a line of $77 \%$ of PPP [47]. The size of extra arch support was $5 \mathrm{~mm}$ thick Lunalastic (NORA Freudenberg GmbH, Weinheim, Germany) in addition to arch support resulted from the casting technique [42]. Casting techniques for custom-insoles making, insole design, and manufacturing processes also have been reported by some studies $[40,47,49,54,55,62]$.

\section{Outcome measures}

Eighteen studies [30, 38-42, 44, 46-55, 64] measured PPP as the primary outcome, and the majority measured this inshoe. Most of the studies [30, 38, 39, 47, 50, 52, 53, 57, 64] used $200 \mathrm{kPa}$ as an upper threshold to classify the intervention as successful offloading the foot. The remaining studies compared a baseline pressure assessment without the intervention to peak pressure reductions with the interventions. PTI and Force Time Integral (FTI) had also been assessed as a parallel outcome measure in some studies [40, 48-51, $53,55]$. Other studies $[50,51,55,64]$ also measured contact area and soft tissue thickness (STT) $[46,48]$ as a parallel outcome. Some single parameters measured by the studies were maximum force, contact area [64], and walking convenience [42]. One study [41] reported foot-sole hardness as an indicator and reduction in shore hardness value. Six studies [56-61] reported ulcer recurrence as a primary outcome measure and another study [62] reported on ulcerative and non-ulcerative lesions as the primary outcome. Three studies $[57,58,60]$ measured patient adherence in their study as a secondary outcome.

The Pedar-X system (Novel GmbH, Germany) was the most commonly used in-shoe plantar pressure measuring device by studies [30, 38-40, 45, 47, 49, 50, 54, 57] followed by the F-Scan system (Tekscan Inc. USA) [42, $44,46,48,53,55]$. Other systems included RS Scan system (RSScan, Ole, Belgium) [51]. Charanya et al. [41] used a pedobarograph system developed by Patil et al. [65-67] to capture the walking foot pressure image and data analysis.

The sensor's thickness of the Pedar-X system is 2 $\mathrm{mm}$ [39, 40], F-Scan $0.18 \mathrm{~mm}$ [55], and RS Scan 0.7 $\mathrm{mm}$ [51]. Both sensors of Pedar-X and F-Scan collect pressure data at $50 \mathrm{~Hz}[44,47]$, and both have four sensors per $\mathrm{cm}^{2}[38,53]$. RS Scan sensors collect data at $500 \mathrm{~Hz}$ [51]. Studies using Pedar-X systems used steps between 20 to $40[40,47,49,54]$ and 10 to 20 $\mathrm{m}$ walk-way [38, 45, 49]. Studies using F-Scan systems used walk-way length between 6.1 to $10 \mathrm{~m}[44,55]$. RS Scan collected dynamic in-shoe pressure data for $8 \mathrm{~s}$ (10-16 steps) [51]. 
Table 2 Study characteristics of selected articles for ulcer recurrence as the primary outcome measure

\begin{tabular}{|c|c|c|c|c|c|c|c|c|}
\hline $\begin{array}{l}\text { Author, } \\
\text { date }\end{array}$ & Location & $\begin{array}{l}\text { Study } \\
\text { design }\end{array}$ & $\begin{array}{l}\text { Follow } \\
\text { up } \\
\text { period }\end{array}$ & $\begin{array}{l}\text { Sample } \\
\text { size }\end{array}$ & $\begin{array}{l}\text { Sample } \\
\text { characteristics }\end{array}$ & $\begin{array}{l}\text { Intervention \& } \\
\text { Comparison }\end{array}$ & $\begin{array}{l}\text { Outcome } \\
\text { measures }\end{array}$ & Result \\
\hline $\begin{array}{l}\text { Busch } \\
\text { et al. } 2003 \\
{[56]}\end{array}$ & Germany & $\begin{array}{l}\text { Prospective } \\
\text { cohort }\end{array}$ & $\begin{array}{l}\text { Up to } \\
42 \\
\text { months }\end{array}$ & 92 & $\begin{array}{l}\text { Diabetes } \\
\text { Neuropathy } \\
\text { Peripheral } \\
\text { vascular } \\
\text { disease (PVD) }\end{array}$ & $\begin{array}{l}\text { Lucro SDS vs non-SDS } \\
\text { standard footwear }\end{array}$ & $\begin{array}{l}\text { Ulcer } \\
\text { recurrence }\end{array}$ & $\begin{array}{l}\text { Annual ulcer recurrence SDS } \\
15 \% \text { vs Non-SDS } 60 \% \text { when } \\
\text { severe foot deformity is non- } \\
\text { existent }\end{array}$ \\
\hline $\begin{array}{l}\text { Bus et al. } \\
2013[57]\end{array}$ & Netherlands & $\mathrm{RCT}$ & $\begin{array}{l}18 \\
\text { months }\end{array}$ & 171 & $\begin{array}{l}\text { Diabetes } \\
\text { Neuropathy } \\
\text { Healed } \\
\text { plantar ulcers }\end{array}$ & $\begin{array}{l}\text { Custom-made footwear } \\
\text { with and without } \\
\text { modifications based on } \\
\text { in-shoe pressure analysis }\end{array}$ & $\begin{array}{l}\text { Ulcer } \\
\text { recurrence } \\
\text { Adherence of } \\
\geq 80 \% \text { steps } \\
\text { taken }\end{array}$ & $\begin{array}{l}\text { Modified custom-made foot- } \\
\text { wear are only useful in off- } \\
\text { loading forefoot area if they } \\
\text { are worn as per advised (Ad- } \\
\text { herence } \geq 80 \% \text { ) }\end{array}$ \\
\hline $\begin{array}{l}\text { Chantelau } \\
\text { et al. } 1990 \\
\text { [58] }\end{array}$ & Germany & $\begin{array}{l}\text { Prospective } \\
\text { cohort }\end{array}$ & $\begin{array}{l}25 \\
\text { months }\end{array}$ & 50 & $\begin{array}{l}\text { Diabetes } \\
\text { Neuropathy } \\
\text { PVD } \\
\text { History of } \\
\text { healed } \\
\text { plantar foot } \\
\text { ulcer } \\
\text { Partial or } \\
\text { forefoot } \\
\text { amputation }\end{array}$ & $\begin{array}{l}\text { Custom-made footwear } \\
\text { with rocker soles and } \\
\text { custom-made insoles } \\
\text { with } 10 \mathrm{~mm} \text { thickness, }\end{array}$ & $\begin{array}{l}\text { Ulcer } \\
\text { recurrence } \\
\text { Adherence } \\
\text { (regular vs } \\
\text { irregular } \\
\text { wearing of } \\
\text { footwear and } \\
\text { insoles) }\end{array}$ & $\begin{array}{l}\text { Regular wearing of footwear } \\
\text { and insoles reduced the } \\
\text { relative risk of foot ulceration } \\
\text { to } 0.48 \text { ( } 95 \% \text { confidence } \\
\text { interval } 0.29 \text { to } 0.79 \text { ), } \\
\text { compared with irregular } \\
\text { wearing }\end{array}$ \\
\hline $\begin{array}{l}\text { Lavery } \\
\text { et al. } 2012 \\
\text { [59] }\end{array}$ & USA & $\mathrm{RCT}$ & $\begin{array}{l}18 \\
\text { months }\end{array}$ & 299 & $\begin{array}{l}\text { Diabetes } \\
\text { Neuropathy } \\
\text { Healed foot } \\
\text { ulcers } \\
\text { Foot } \\
\text { deformity }\end{array}$ & $\begin{array}{l}\text { Shear reducing insole } \\
\text { (SRI) with standard } \\
\text { therapy group (STG) } \\
\text { with therapeutic } \\
\text { footwear, diabetic foot } \\
\text { education and care }\end{array}$ & $\begin{array}{l}\text { Ulcer } \\
\text { recurrence }\end{array}$ & $\begin{array}{l}\text { SRI group were } 3.5 \text { times less } \\
\text { likely to develop foot ulcers } \\
\text { comparing to the STG group. } \\
\text { No significant difference in } \\
\text { the frequency of footwear } \\
\text { and insole usage in SRI or } \\
\text { STG group. }\end{array}$ \\
\hline $\begin{array}{l}\text { López- } \\
\text { Moral } \\
\text { et al. } 2019 \\
\text { [60] }\end{array}$ & Italy & $\mathrm{RCT}$ & $\begin{array}{l}18 \\
\text { months }\end{array}$ & 51 & $\begin{array}{l}\text { Diabetes } \\
\text { Neuropathy } \\
\text { Healed } \\
\text { plantar ulcers }\end{array}$ & $\begin{array}{l}\text { Semi-rigid (control) and } \\
\text { rigid rocker sole (test) } \\
\text { therapeutic footwear }\end{array}$ & $\begin{array}{l}\text { Ulcer } \\
\text { recurrence } \\
\text { Adherence > } \\
60 \%\end{array}$ & $\begin{array}{l}\text { Rigid rocker sole can reduce } \\
\text { risk of re-ulceration at fore- } \\
\text { foot by } 64 \% \text { compared to } \\
\text { semi-rigid rocker sole }\end{array}$ \\
\hline $\begin{array}{l}\text { Rizzo et al. } \\
2012 \text { [61] }\end{array}$ & Italy & $\mathrm{RCT}$ & 5 years & 298 & $\begin{array}{l}\text { Diabetes } \\
\text { Neuropathy } \\
\text { Healed } \\
\text { plantar foot } \\
\text { ulcer } \\
\text { Minor } \\
\text { amputation }\end{array}$ & $\begin{array}{l}\text { Standard comfort } \\
\text { footwear vs custom } \\
\text { insoles and footwear as } \\
\text { per Dahmen et al. } \\
\text { algorithm }\end{array}$ & $\begin{array}{l}\text { Ulcer } \\
\text { recurrence }\end{array}$ & $\begin{array}{l}\text { Ulcer recurrence rates in } 1,3 \\
\& 5 \text { years are } 11.5 \% \text { vs } 38.6, \\
17.6 \% \text { vs } 61,23.5 \% \text { vs } 72 \% \\
\text { where forefoot deformities } \\
\text { are predominant among the } \\
\text { participants. }\end{array}$ \\
\hline $\begin{array}{l}\text { Ulbrecht } \\
\text { et al. } 2014 \\
\text { [62] }\end{array}$ & USA & $\mathrm{RCT}$ & $\begin{array}{l}15 \\
\text { months }\end{array}$ & 150 & $\begin{array}{l}\text { Diabetes } \\
\text { Neuropathy } \\
\text { Healed } \\
\text { plantar foot } \\
\text { ulcer (MTHs) } \\
\text { Increased } \\
\text { barefoot } \\
\text { plantar } \\
\text { pressure }\end{array}$ & $\begin{array}{l}\text { Control: Standard } \\
\text { custom-made insoles } \\
\text { from three different } \\
\text { suppliers } \\
\text { Experimental: Insoles } \\
\text { made according to the } \\
\text { protocol in Owings et al. } \\
2008 .\end{array}$ & $\begin{array}{l}\text { Ulcerative or } \\
\text { non-ulcerative } \\
\text { lesions at the } \\
\text { plantar fore- } \\
\text { foot in MTHs } \\
\text { regions }\end{array}$ & $\begin{array}{l}\text { Foot shape and plantar } \\
\text { pressure-based custom in- } \\
\text { soles provide superior offload- } \\
\text { ing than insoles made from } \\
\text { foot shape and clinical } \\
\text { insights. }\end{array}$ \\
\hline
\end{tabular}

MP Metatarsal Pad, MB Metatarsal Bar, MD Metatarsal Dome, SDS Stock Diabetic Shoes, MTH1 First Metatarsal Head, FTI Force Time Integral, PTI Pressure Time Integral, MPP Mean Peak Pressure, TCI Total Contact Insoles, SRI Shear Reducing Insoles, STG Standard Therapy Group

\section{Reductions in forefoot plantar pressure}

Arts et al. [38] reported on the effectiveness of footwear and insole design based on the algorithm proposed by Dahmen et al. [68]. The rate of pressure reduction was lower at the metatarsals area (29-50\%) compared to midfoot (81\%) and known ulcer location (62\%) [38] when footwear and insoles are designed according to Dahmen's algorithm.
Sole design (rocker sole) was the most reported design feature and some reported on detailed configurations such as rocker apex position [30, 38, 41, 50-52, $56,61]$, rocker apex angle [52], rocker angle [30, 51, $52,60]$, rigidity or hardness $[30,38,41,42,53,56$, $60,61]$ and, material type $[41,50,51,60,61]$. A rocker sole configuration with apex position at $52 \%$ of the footwear length, $20^{\circ}$ rocker angle, and $95^{\circ}$ apex 
angle can yield peak pressure $<200 \mathrm{kPa}$ in $71-81 \%$ cases [52].

Some studies reported on footwear upper design features, such as upper height (high footwear $16 \mathrm{~cm}$, Bottine $12.5 \mathrm{~cm}$, Low footwear $(6.5 \mathrm{~cm})$ [38, 51, 61], footwear depth [39, 50, 56, 57, 60, 64], leg and tongue profile $[38,57,61]$. Other design features are; upper material, collar, lining, toe puff $[50,56,60]$, heel counter, fastening system [53,60] and active heel height [51].

Non-weight-bearing (NWB) casting technique yields more effective custom-made insoles to offload the hallux region and semi-weight-bearing (SWB) casting technique is more effective to offload 1-3 metatarsal heads (MTHs) [55]. The NWB insoles also yield the highest arch support comparing to insoles made by other casting techniques [55].

Insoles designed based on foot shape and plantar pressure data are more effective to offload the forefoot region compared to insoles designed based on foot shape only $[49,54,62]$. The outcome can be between 32 to $21 \%$ improvement from shape-only and traditionally manufactured insoles out of polypropylene base [49].

Custom-made insoles with multi-density, softer materials have demonstrated improved forefoot offloading compared to higher-density EVA ( $55^{\circ}$ shore A). Extra arch support, metatarsal pads, a plastazote top cover, and local cushioning can further reduce plantar forefoot pressure [42, 64]. Metatarsal pad, local cushion and a plastazote top cover can reduce peak pressure by 14 to $15.9 \%$ on their own. A plastazote top cover combined with a metatarsal pad and local cushioning reduces 24 and 22\% PPP at the forefoot [39].

\section{Reductions in ulcer recurrence}

López-Moral et al. [60] explored the effect of two rocker soles: semi-rigid (Wellwalk technology with Vibram Strips) and rigid on the recurrence of ulceration. By using, a rigid rocker sole the risk of re-ulceration at the forefoot was reduced by $64 \%$ when compared with semirigid rocker sole footwear.

Busch et al. [56] examined the effect of two different footwear (Lucro stock diabetic footwear versus regular retail footwear) with insoles on ulcer relapse of 92 participants with high-risk neuropathic feet at 12 and 42 months. The footwear was available in three different widths with differing features: rocker bottom outsoles and soft upper with three layers. This combined footwear and insoles reduced ulcer relapse by $45 \%$ compared with standard footwear within the first year.

Rizzo et al. [61] compared a treatment group who were given therapeutic footwear designed as per Dahmen et al. [57, 68] and custom-made insoles to a control group who received standard footwear. The participants were assessed for ulcer occurrence and relapse at 12, 36 and 60 months. Ulcer relapse rates were significantly lower $(11.5 \%$ versus $38.6 \%$ at 12 months, $17.6 \%$ versus $61 \%$ at 36 months and $23.5 \%$ versus $72 \%$ at 60 months) in the treatment group than controls.

Lavery et al. [59] examined the effect of shearreducing insoles on ulcer recurrence when compared with standard insoles in the same style of footwear. Shear-reducing insoles were 3.5 times less likely to create ulcers in the study participants compared to the standard insoles, although, both insole types demonstrated equivalent plantar pressure reduction [69].

In another study [57] based on the algorithm proposed by Dahmen et al. $[57,68]$ the treatment group received custom-made footwear that was adjusted following inshoe pressure analysis. Controls received custom-made footwear without the in-shoe pressure analysis. The primary outcome was ulcer relapse after 18 months. The outcomes were not significantly different due, in part, to variance in patient adherence.

\section{Discussion}

Footwear and insoles are complex biomechanical interventions due to variance in design, materials, manufacturing methods, individual preferences and rates of adherence. This complexity is compounded when it is considered alongside the range of foot pathologies that co-exist with diabetes. Forefoot structural deformities are prevalent in this patient group [11, 12] increasing inshoe plantar pressure at the metatarsal heads. The importance of footwear and insoles in offloading PPP for preventing plantar forefoot foot ulceration is well documented [70, 71]. However, the specifications of design parameters and materials that can reduce PPP at the forefoot area are not precise. Reduction of PPP is one of the major factors to reduce the risk of ulcer occurrence and recurrence. This review explores the identification of critical design features and materials used in footwear and insole manufacturing that can reduce PPP at the forefoot and prevent ulcer occurrence and recurrence. Summery of those features that are available in the literature has been presented in Appendix 1 and 2.

Several studies have suggested rocker sole profile as the most recommended design to offload PPP at the forefoot $[30,39,51,52,56,60,61]$. The studies showed strong evidence for the rocker sole with evidence pointing towards specific variations of the rocker sole: such as apex position, apex angle, rocker angle and rigidity of sole materials. An RCT [60] showed that a rocker sole configuration with the pivot point under the metatarsal heads and rigid sole materials improve plantar pressure offloading at the forefoot compared to rocker sole made with semi-rigid materials. In a 6 month follow-up, the plantar ulcer recurrence rate was 23 and 64\% among the experimental and control group where sole rigidity was the only variant. Preece et al. [52] and Praet et al. [51] 
compared apex position and rocker angle for rocker sole design in their studies. They recommended an apex position at $52-63 \%$ of shoe length and rocker angle of $20-23^{0}$ to provide effective offloading at the forefoot $(<200 \mathrm{kPa})$, finding it more effective than any other lower or higher values of those respective parameters.

Arts et al. [38] in the Netherlands and Rizzo et al. [61] in Italy tested the effect of footwear design suggested by the consensus-based algorithm proposed by Dahmen et al. [68]. The key footwear design features in Dahmen algorithm are based on medical conditions. For example, the recommendations for a person with diabetes and history of neuropathic ulcers are footwear with a high upper (above ankle boots), stiffened tongue and leg uppers, rigid rocker soles with early pivot point. Both studies used above-ankle boots with custom-made insoles to offload pressure at the forefoot area. Both studies found that footwear and insoles designed according to this algorithm, are effective in offloading the neuropathic diabetic foot. However, Arts and colleagues [38] found that the algorithm is not as effective for footwear specifications to offload plantar pressure at the metatarsal heads.

There is a lack of guidance in the literature on footwear modifications that offload the forefoot. Footwear modification (also known as footwear customisation or optimisation) is common in both prefabricated and fully custom-made footwear. Most frequent footwear modifications are a re-configuration of rocker sole profile, such as early or significant pivot point (rocker angle) and stiffening the outer sole [30, 39]. Footwear modification success $(\leq 200 \mathrm{kPa})$ is least at the forefoot [38,39]. Bus et al. [30] recommended in-shoe plantar pressure analysis as an effective tool to guide the modifications for offloading the target regions in the neuropathic foot.

Insole modification features include local cushioning, replacing top covers with plastazote and applying a new or re-positioning existing metatarsal bars and metatarsal domes [30, 39, 47, 61], removing plugs, and adding arch supports $[61,64]$. These are the most effective (PPP reduced $\leq 200 \mathrm{kPa}$ ) modifications in offloading or reducing PPP in targeted regions [30, 39]. The targeted regions were determined by the history of ulceration or from PPP measurements data. These modifications in the insole are proven to be effective in offloading plantar pressure at an optimal level. However, they are least effective in offloading pressure at the metatarsal heads [38, 39].

Pedorthists commonly use a higher upper height in their treatment of neuropathic forefoot ulcers. Dahmen et al. [68] and Diabetic Foot Australia (DFA) guideline [34] support such practice. However, Praet et al. [51] showed that high-ankle boots did not influence plantar pressure offloading when compared with low cut footwear. The authors suggest that although high-ankle boots do not change plantar pressures, they may reduce shear forces inside the shoe at the forefoot by increasing contact area around the ankle. Considering these findings, further studies assessing high-ankle boots will help to inform clinicians working in this field.

Many design features were not examined in the literature. Higher quality research is required to scientifically examine other important footwear design parameters, including heel height, toe height, upper materials, sole materials, heel counters, and closure systems for this therapeutic target.

There was moderate evidence [72] to suggest using total contact insoles $[49,55,61,62]$, metatarsal pads $[40,44,46,48,62]$, metatarsal bars $[47,61]$ and plastazote top covers [39] to reduce PPP. Arts et al. [39] recommended plastazote as a top cover over leather due to its superiority in peak pressure offloading, but they need to be replaced every 6 months. Two studies $[50,53]$ also included prefabricated insoles as interventions, which also showed a reduction in forefoot plantar pressure.

In practice, the use of custom-made insoles over prefabricated devices needs to be considered in relation to cost versus benefit. Paton and colleagues [50] used two different insoles, made out of EVA and Poron, and compared cost as well. Custom devices were $18 \%$ higher cost in delivery than prefabricated insoles. The main difference was where the foot was cast to make the insoles, or insoles were selected from stock. There was no significant difference in PPP reduction between the two types of insoles. Custom-made insoles were, however, found to reduce PTI more than prefabricated insoles and lasted longer [50]. Customised devices may be preferred in practice as they account for structural changes in the diabetic foot, which is likely the reason that they reduce PTI more than prefabricated devices. Other studies [30, $40,46,48,53,55]$ that compared PPP reduction capacity of the custom-made insoles with prefabricated insoles and not examined the cost, those found custom-made insoles to be more effective in pressure offloading in almost every region of the foot.

Most common insole base materials are EVA with the hardness of $50-55^{\circ}$ Shore A and $30-35^{\circ}$ Shore A [47, 53] and the latter material showed improved performance in offloading PPP. However, the medium-density EVA base (30-35 $5^{\circ}$ Shore A) insoles need more frequent replacement than the higher density EVA group insoles due to material fatigue.

PPT or Poron as mid-layer [56] and top cover materials either MCR, plastozote or microfiber are effective in plantar forefoot pressure offloading. PPT or Poron is also used as a top cover in some insole designs $[56,64]$. Use of a leather top cover is of limited benefit due to its poor pressure reduction capacity [39]. 
None of the studies looked at the prevention of initial neuropathic plantar forefoot ulcer occurrence rather than a subsequent recurrence ulcer. Additionally, studies did not assess forefoot ulceration in isolation, but whole foot ulceration. PPP reduction in different regions requires different types of offloading. Further, different footwear and insole design features show differences in pressure reduction efficacy in different regions of the foot. The articles relied on in-shoe plantar pressure measurement data as a predictor of ulceration. However, other factors such as co-morbidity and lack of adherence to treatment also contribute to ulcer occurrence.

Plantar tissue stress incorporates vertical plantar pressure, horizontal shear pressure, and the frequency at which it is applied [73]. The reliance on plantar pressures as a predictor of ulceration may, therefore, be only one part of the picture. Lavery et al. [59, 69] reported that two different insoles (shear-reducing and standard insoles) with equivalent plantar pressure reduction capacity could have a significantly different outcome in ulcer recurrence where shear-reducing is the only differentiation factor. Shear-reducing insoles had 3.5 times higher ulcer prevention capacity than the standard insoles in the study participants. Since design features are likely to influence footwear function, and therefore, adherence, it is important to consider which features may prevent ulceration.

There is limited data in the literature to determine the efficacy of footwear in preventing ulcer occurrence. Preece et al. [52] and Martinez-Santos et al. [47] explored the efficacy of footwear and insole design features, but could not make any recommendations for preventing ulcer occurrence.

In this review, the articles were excluded if the participants had heel ulcer, Charcot foot or any active, dorsal foot ulcers, and these might limit the representation of complete diabetic foot conditions. This may limit the footwear and insole feature recommendations for those feet that have those conditions.

Heterogeneity in study designs, interventions, outcome measures and footwear and insoles design features make it also very difficult to come into a conclusion. Greater variations in participant's inclusion criteria and foot deformities, footwear and insole types, their measuring, casting and designing techniques, in-shoe pressure analysis systems may result in inconsistent data. Hence, we can not make a clear comparison or pool data to analyse further.

Because of the need to customise to the individual, the success of custom-made footwear as an intervention in offloading the plantar foot is dependent on the knowledge and skills of the prescribers and manufacturers $[30,40,55]$. The studies in this review used a variety of skilled practitioners in these roles such as orthopaedic shoemakers, pedorthists depending on the region. The presence of these practitioners in the interdisciplinary team approach in high-risk foot services is increasingly recognised ([34], http://nadc. net.au/foot-network/).

Several studies [30, 42, 50, 57, 60, 61] explored patient satisfaction and adherence to wearing footwear and insoles. Patient adherence to wearing therapeutic footwear is vital to ensure improved offloading and ulcer prevention $[57,60,61]$. No difference was found in patients' perceptions of custom-made versus prefabricated insoles [50]. Adding arch support and large metatarsal domes to basic insoles reduces patient adherence and walking comfort, despite evidence that these features improve pressure offloading [42].

Studies did not report the factors that influence adherence to therapy, which also limits the application of our findings. Consideration of patient expectations, effective education on footwear and activity-specific device designs are limited in the literature. Studies also did not consider geographical and socioeconomic factors. Most studies [30, 38, 39, 42, 47, 48, 50-53, $56,57,60,61]$ were carried out in developed countries [63] with climates conducive to using ankle-high boots. Also, the practicality of these ankle-high boots for countries with warmer climates needs revisiting concerning patient adherence.

There was no study to take a personalised-treatment approach to focus on an individual's need or preference to increase adherence. Footwear is a very personal item, and a pre-study participant's feedback on their future footwear is crucial as opposed to only post-study feedback as adherence plays a vital role in an individual's outcome [51, 57, 58, 60]. Study designs like the N-of-1 or single-patient-trial design $[74,75]$ may bridge the gap in the literature.

Appropriate footwear design that takes into consideration the needs of low-income countries and those with warmer climates are limited in the literature, even though the prevalence of diabetes tend to be higher among the populations in these regions [76].

\section{Conclusion}

There is limited evidence to inform footwear and insole interventions, especially in conjunction with inshoe plantar pressure reduction. The available evidence supports the identification of footwear and insole design and modification parameters that can influence forefoot plantar pressure reduction. Prevention of ulcer occurrence or recurrence at the plantar forefoot region in diabetic patients is limited. Further research is needed to improve care for people with diabetic foot ulceration. 


\section{Appendix 1}

Table 3 Description of footwear features designed to reduce neuropathic forefoot plantar ulcer occurrence found in the literature

The description provided on footwear upper and sole design

Bottine $(12.5 \mathrm{~cm})$ or high footwear $(16 \mathrm{~cm})$ for upper height

The toughened outsole, resilient material on the heel

Toughened leg and tongue

Rocker profile outsole with early and normal pivot point

Fully custom-made orthopaedic footwear and semi-custom (extra depth + width off-the-shelf footwear)

Thin, seamless cotton socks

Lucro stock diabetic footwear (SDS) with toughened outer-sole with forefoot rocker

Fully custom footwear manufactured with features of Ankle-high footwear, stiffened rubber outsole with rocker bottom sole. Modification: Outsole rocker pivot point relocation and rocker angle

Toughened rocker profile rubber outsole, shoes or sandals with smooth leather, adjustable front and back straps for sandals or closed in footwear

Van Lier ${ }^{\oplus}$, Netherlands, Outer sole shore type A: 86

Standard diabetic footwear (extra depth leather footwear, Dr. Foot Technology Co.,)

Semi-rigid rocker sole (Wellwalk technology with Vibram Strips) and rigid rocker sole (reinforced with composite fibre). The rocker sole was anteroposterior rocker and pivot point behind the metatarsal heads with $20^{\circ}$ rocker angle. The shoes had rigid heel counter, extra depth toe boxes (14 to $16 \mathrm{~mm}$ deeper than standard shoes), lace or buckle closures.

SoleTech new footwear, style E3010

Modular non-bespoke diabetic footwear with soft leather upper, plain vamp, secure fastening, microfibre lining material, padded collar, wall toe puff, EVA micro rubber sole unit with rocker where the apex is posterior to metatarsophalangeal joints line (County Orthopedic Footwear Ltd).

Eight types of rocker sole configuration by two types of rocker angle $15^{\circ} \& 20^{\circ}$ each for the apex positions of 52,57 , $62,67 \%$ of footwear length.

(Duna, Italy)

Semi-rigid outer sole or stiff rocker sole, a stable heel counter, and adjustable laces or Velcro straps Study(s)

Arts et al., 2012 [38],

Bus et al. 2013 [57]

Preece et al. 2017 [52]

Rizzo et al. 2012 [61]

Arts et al. 2015 [39]

Busch et al. 2003 [56]

Bus et al. 2011 [30]

Charanya et al. 2004 [41]

Guldemond et al. 2007 [42]

Lin et al. 2013 [64]

López-Moral et al. 2019 [60]

Mueller et al. 2006 [48]

Paton et al. 2012 [50]

Preece et al. 2017 [52]

Tang et al. 2014 [53]

Arts et al., 2012 [38]

Arts et al. 2015 [39]

Most frequent single modifications are replacement top cover of the insole, local cushioning of the insole, the addition of pad to the insole.

Combined modification of insole: Above items and removal of local materials as an addition.

Flat insoles with rear base: $42^{\circ}$ Shore hardness and anterior base $20^{\circ}$ Shore hardness $6 \mathrm{~mm}$ thick Lunasoft ${ }^{\oplus}$ and $3 \mathrm{~mm}$ overall top-cover of PPT with $17^{\circ}$ Shore A hardness.

Busch et al. 2003 [56]

\section{Appendix 2}

Table 4 Description of insole features designed to reduce neuropathic forefoot plantar ulcer occurrence found in the literature

\begin{tabular}{ll}
\hline The description provided on insole design & Study(s)
\end{tabular}

Fully custom-made insoles with multi-density and multi-layered materials, an open-cell or cross cell material top cover. Modification: Local removal of material on the insole, local softening, adding metatarsal, hallux pad or bar on the insole, Bus et al. 2011 [30] replacement of the top cover

Custom made insole made from multilayered materials with cork base added with micro cork, a mid-layer of EVA base multiform. Additional metatarsal pad or bar with extra arch support.

Bus et al. 2013 [57]

Insole made of $12 \mathrm{~mm}$ microcellular rubber (MCR), shore value $20^{\circ}$

Charanya et al. 2004 [41]

Metatarsal dome, arch supports, and extra arch supports

Insoles made of $5 \mathrm{~mm}$ Lunalastic as the top layer, $8 \mathrm{~mm}$ Lunasoft SL as the bottom layer, $1.1 \mathrm{~mm}$ Rhenoflex $3208^{\oplus}$ as

internal reinforcement. Every layer of arch support has $5 \mathrm{~mm}$ thickness of Lunalastic material.

$3 \mathrm{~mm}$ Shore A $35^{\circ}$ EVA as 1 st layer, $2 \mathrm{~mm}$ Velcro and velvet in 2 nd layer and $6 \mathrm{~mm}$ Shore A $50^{\circ}$ Poron in the third layer

Multilayered with $40^{\circ}$ shore hardness EVA base and Poron top cover, cut-out in the affected metatarsal head.

Insole base with $5 \mathrm{~mm} 50^{\circ}$ Shore A EVA with three different metatarsal bar (MB) positioning out of two different types materials: $20^{\circ}$ Shore A EVA, $20^{\circ}$ Shore A Poron

$1.27 \mathrm{~cm}$ thick number 2 plastazote with shore value approx. 35, metatarsal pad (MP), positioned proximal to metatarsal heads

Guldemond et al. 2007 [42]

Full length $3 \mathrm{~mm}$ blue medium density Ethylene Vinyl Acetate (EVA) shell and $6 \mathrm{~mm}$ grey Poron top cover

SM-2, 3 \& 4: 3/4 custom insoles with EVA base and $3 \mathrm{~mm}$ PPT full-length top cover

$\mathrm{SM}-5$ \& 6: Custom insoles with EVA base and $3 \mathrm{~mm}$ PPT full-length top cover

Insoles from the static footprint and foam box impression, configured with arch support, metatarsal bar, soft fillers. Insole materials: PPT, Duuroterm, Alcaform

Custom insoles: $35 \& 55^{\circ}$ Shore A hardness EVA (14 mm thickness) for custom made insoles manufactured from positive plaster moulds, metatarsal bars proximal to II-IV MTH's.

Prefabricated insole: Hardcore EVA base, $12^{0}$ Shore hardness microfiber top layer (GloboTec ${ }^{\oplus}$ comfort 312,750,501,400)

Lin et al. 2013 [64]

López-Moral et al. 2019 [60]

Martinez-Santos et al. 2019 [47]

Mueller et al. 2006 [48]

Paton et al. 2012 [50]

Praet et al. 2003 [51]

Rizzo et al. 2012 [61]

Tang et al. 2014 [53] 


\section{Supplementary information}

Supplementary information accompanies this paper at https://doi.org/10. 1186/s13047-020-00400-4.

Additional file 1. Search term and strategy.

Additional file 2. Quality assessment of the included articles.

\section{Abbreviations}

RCT: Randomised controlled trial; PRISMA: Preferred reporting items for systematic reviews and meta-analysis; BMI: Body mass index; DPN: Diabetic peripheral neuropathy; PPP: Peak plantar pressure; PTI: Pressure time integral; STT: Soft tissue thickness; DFA: Diabetic foot Australia; HRFS: High-risk foot services

\section{Acknowledgements}

Not applicable.

\section{Authors' contributions}

The search strategy was performed by SA. Data extraction was performed by SA and SN. Quality analysis was performed by SA. All authors provided input into the review draft and agreed on the final manuscript. All authors read and approved the final manuscript.

\section{Funding}

Not applicable.

\section{Availability of data and materials}

The author can be contacted for any data requests.

\section{Ethics approval and consent to participate}

Not applicable.

\section{Consent for publication}

Not applicable.

\section{Competing interests}

The authors declare that they have no competing interests.

Received: 10 February 2020 Accepted: 22 May 2020

Published online: 04 June 2020

\section{References}

1. Boulton AJ, Vileikyte L, Ragnarson-Tennvall G, Apelqvist J. The global burden of diabetic foot disease. Lancet. 2005;366:1719.

2. Waaijman $R$, de Haart $M$, Arts ML, Wever $D$, Verlouw AJ, Nollet F, Bus SA. Risk factors for plantar foot ulcer recurrence in neuropathic diabetic patients. Diabetes Care. 2014;37:1697.

3. Ghanassia E, Villon L, dit Dieudonné J-FT, Boegner C, Avignon A, Sultan A. Long-term outcome and disability of diabetic patients hospitalized for diabetic foot ulcers: a 6.5-year follow-up study. Diabetes Care. 2008;31:1288.

4. Peters EJ, Armstrong DG, Lavery LA. Risk factors for recurrent diabetic foot ulcers: site matters. Diabetes Care. 2007:30:2077.

5. Pound N, Chipchase S, Treece K, Game F, Jeffcoate W. Ulcer-free survival following management of foot ulcers in diabetes. Diabet Med. 2005;22:1306.

6. Molines-Barroso R, Lázaro-Martínez J, Aragón-Sánchez J, García-Morales E, Beneit-Montesinos J, Álvaro-Afonso F. Analysis of transfer lesions in patients who underwent surgery for diabetic foot ulcers located on the plantar aspect of the metatarsal heads. Diabet Med. 2013;30:973.

7. Armstrong DG, Boulton AJ, Bus SA. Diabetic foot ulcers and their recurrence. N Engl J Med. 2017;376:2367.

8. Levin ME, O'Neal L. The diabetic foot: pathophysiology, evaluation, and treatment. Diabetic Foot. 1988;1:1-50.

9. Lazzarini PA, O'Rourke SR, Russell AW, Derhy PH, Kamp MC. Reduced incidence of foot-related hospitalisation and amputation amongst persons with diabetes in Queensland, Australia. PLoS One. 2015:10:e0130609.

10. Cavanagh P, Boulton A, Sheehan P, Ulbrecht J, Caputo G, Armstrong D, Chantelau E, Reiber G, Smith D, Heagerty P. Letters-therapeutic footwear in patients with diabetes. JAMA. 2002;288:1229.
11. Lázaro-Martínez UL, Aragón-Sánchez J, Álvaro-Afonso FJ, García-Morales E, García-Álvarez Y, Molines-Barroso RJ. The best way to reduce Reulcerations:if you understand biomechanics of the diabetic foot, you can do it. Int J Low Extrem Wounds. 2014;13:294.

12. Bus SA. Foot structure and footwear prescription in diabetes mellitus. Diabetes Metab Res Rev. 2008;24:590.

13. Reiber GE, Vileikyte L, Ed B, Del Aguila M, Smith DG, Lavery LA, Boulton A Causal pathways for incident lower-extremity ulcers in patients with diabetes from two settings Diabetes Care. 1999;22(1):157-62.

14. Carls GS, Gibson TB, Driver VR, Wrobel JS, Garoufalis MG, DeFrancis RR, Wang S, Bagalman JE, Christina JR. The economic value of specialized lower-extremity medical care by podiatric physicians in the treatment of diabetic foot ulcers. J Am Podiatr Med Assoc. 2011:101:93.

15. van Schie $\mathrm{CH}$. Neuropathy: mobility and quality of life. Diabetes Metab Res Rev. 2008:24:S45.

16. Singh N, Armstrong DG, Lipsky BA. Preventing foot ulcers in patients with diabetes. JAMA. 2005;293:217.

17. Hidmark A, Fleming T, Vittas S, Mendler M, Deshpande D, Groener J, Müller B, Reeh P, Sauer S, Pham M. A new paradigm to understand and treat diabetic neuropathy. Exp Clin Endocrinol Diabetes. 2014;226:201.

18. Fernando $M$, Crowther $R$, Lazzarini $P$, Sangla $K$, Cunningham M, Buttner $P$, Golledge J. Biomechanical characteristics of peripheral diabetic neuropathy: a systematic review and meta-analysis of findings from the gait cycle, muscle activity and dynamic barefoot plantar pressure. Clin Biomech. 2013; 28.831.

19. Sawacha Z, Spolaor F, Guarneri G, Contessa P, Carraro E, Venturin A, Avogaro A, Cobelli C. Abnormal muscle activation during gait in diabetes patients with and without neuropathy. Gait \& Posture. 2012;35:101.

20. Ko S-U, Stenholm S, Chia CW, Simonsick EM, Ferrucci L. Gait pattern alterations in older adults associated with type 2 diabetes in the absence of peripheral neuropathy—results from the Baltimore Longitudinal Study of Aging. Gait Posture. 2011;34:548.

21. Guiotto A, Sawacha Z, Guarneri G, Cristoferi G, Avogaro A, Cobelli C. The role of foot morphology on foot function in diabetic subjects with or without neuropathy. Gait Posture. 2013;37:603.

22. Chen WM, Lee T, Lee PV, Lee JW, Lee SJ. Effects of internal stress concentrations in plantar soft-tissue--a preliminary three-dimensional finite element analysis. Med Eng Phys. 2010;32:324.

23. Chao CY, Zheng Y-P, Cheing GL. Epidermal thickness and biomechanical properties of plantar tissues in diabetic foot. Ultrasound Med Biol. 2011;37 1029

24. Pai S, Ledoux WR. The compressive mechanical properties of diabetic and non-diabetic plantar soft tissue. J Biomech. 2010;43:1754.

25. Chapman J. "Improving the design of the curved rocker shoe for people with diabetes" (PhD, University of Salford, 2014), 253.

26. van Netten JJ, van Baal JG, Bril A, Wissink M, Bus SA. An exploratory study on differences in cumulative plantar tissue stress between healing and non-healing plantar neuropathic diabetic foot ulcers. Clin Biomech. 2018;53:86.

27. Cavanagh PR, Ulbrecht JS. Clinical plantar pressure measurement in diabetes: rationale and methodology. Foot. 1994;4:123.

28. Bennetts CJ, Owings TM, Erdemir A, Botek G, Cavanagh PR. Clustering and classification of regional peak plantar pressures of diabetic feet. J Biomech 2013;46:19.

29. Rao S, Saltzman CL, Yack HJ. Relationships between segmental foot mobility and plantar loading in individuals with and without diabetes and neuropathy. Gait Posture. 2010;31:251

30. Bus SA, Haspels ROB, Busch-Westbroek TE. Evaluation and optimization of therapeutic footwear for neuropathic diabetic foot patients using in-shoe plantar pressure analysis. Diabetes Care. 2011;34:1595.

31. Bus SA. Priorities in offloading the diabetic foot. Diabetes Metab Res Rev. 2012;28:54.

32. Jeffcoate WJ, Harding KG. Diabetic foot ulcers, Lancet. 2003:361:1545.

33. Collings R, Freeman J, Latour JM, Glasser S, Paton J. Footwear and insole design features to prevent foot ulceration in people with diabetes: a systematic review protocol. JBI Database System Rev Implement Rep. 2017; $15 \cdot 1824$

34. van Netten JJ, Lazzarini PA, Armstrong DG, Bus SA, Fitridge R, Harding K, Kinnear E, Malone M, Menz HB, Perrin BM. Diabetic foot Australia guideline on footwear for people with diabetes. J Foot Ankle Res. 2018;11:2. 
35. Bus SA, Lavery LA, Monteiro-Soares M, Rasmussen A, Raspovic A, Sacco IC, van Netten JJ. Guidelines on the prevention of foot ulcers in persons with diabetes (IWGDF 2019 update). Diabetes Metab Res Rev. 2020;36(S1):e3269.

36. Moher D, Liberati A, Tetzlaff J, Altman DG, Group P. Preferred reporting items for systematic reviews and meta-analyses: the PRISMA statement. PLoS Med. 2009;6:e1000097.

37. Law M, Stewart D, Letts L, Pollock N, Bosch J, Westmorland M. Guidelines for critical review of qualitative studies. McMaster University Occupational Therapy Evidence-Based Practice Research Group, 1998.

38. Arts M, Waaijman R, de Haart M, Keukenkamp R, Nollet F, Bus S. Offloading effect of therapeutic footwear in patients with diabetic neuropathy at high risk for plantar foot ulceration. Diabet Med. 2012;29:1534

39. Arts M, de Haart M, Waaijman R, Dahmen R, Berendsen H, Nollet F, Bus S. Data-driven directions for effective footwear provision for the high-risk diabetic foot. Diabet Med. 2015;32:790.

40. Bus SA, Ulbrecht JS, Cavanagh PR. Pressure relief and load redistribution by custom-made insoles in diabetic patients with neuropathy and foot deformity. Clin Biomech. 2004;19:629.

41. Charanya G, Patil K, Narayanamurthy V, Parivalavan R, Visvanathan K. Effect of foot sole hardness, thickness and footwear on foot pressure distribution parameters in diabetic neuropathy. Proc Inst Mech Eng H J Eng Med. 2004; 218:431.

42. Guldemond N, Leffers P, Schaper N, Sanders A, Nieman F, Willems $P$, Walenkamp $G$. The effects of insole configurations on forefoot plantar pressure and walking convenience in diabetic patients with neuropathic feet. Clin Biomech. 2007;22:81.

43. Donaghue VM, Sarnow MR, Giurini JM, Chrzan JS, Habershaw GM, Veves A. Longitudinal in-shoe foot pressure relief achieved by specially designed footwear in high risk diabetic patients. Diabetes Res Clin Pract. 1996;31:109.

44. Hastings MK, Mueller MJ, Pilgram TK, Lott DJ, Commean PK, Johnson JE. Effect of metatarsal pad placement on plantar pressure in people with diabetes mellitus and peripheral neuropathy. Foot Ankle Int. 2007;28:84.

45. Tung-Liang L, Huey-Min S, Chin-Teng C, Sai-Wei Y, Shih-Yi L, Hong-Ji L, Chung-Yu C, Chan IC, Hsu-Sheng S, Wayne H-HS. The effect of removing plugs and adding arch support to foam based insoles on plantar pressures in people with diabetic peripheral neuropathy. J Foot Ankle Res. 2013;6:1.

46. Lott DJ, Hastings MK, Commean PK, Smith KE, Mueller MJ. Effect of footwear and orthotic devices on stress reduction and soft tissue strain of the neuropathic foot. Clin Biomech. 2007;22:352.

47. Martinez-Santos A, Preece S, Nester CJ. Evaluation of orthotic insoles for people with diabetes who are at-risk of first ulceration. J Foot Ankle Res. 2019;12:35.

48. Mueller MJ, Lott DJ, Hastings MK, Commean PK, Smith KE, Pilgram TK. Efficacy and mechanism of orthotic devices to unload metatarsal heads in people with diabetes and a history of plantar ulcers. Phys Ther. 2006;86:833.

49. Owings TM, Woerner JL, Frampton JD, Cavanagh PR, Botek G. Custom therapeutic insoles based on both foot shape and plantar pressure measurement provide enhanced pressure relief. Diabetes Care. 2008;31:839.

50. Paton JS, Stenhouse EA, Bruce G, Zahra D, Jones RB. A comparison of customised and prefabricated insoles to reduce risk factors for neuropathic diabetic foot ulceration: a participant-blinded randomised controlled trial. J Foot Ankle Res. 2012;5:31.

51. Praet SF, Louwerens J-WK. The influence of shoe design on plantar pressures in neuropathic feet. Diabetes Care. 2003;26:441.

52. Preece SJ, Chapman JD, Braunstein B, Brüggemann G-P, Nester CJ. Optimisation of rocker sole footwear for prevention of first plantar ulcer: comparison of group-optimised and individually-selected footwear designs. J Foot Ankle Research. 2017;10:27

53. Hellstrand Tang U, Zügner R, Lisovskaja V, Karlsson J, Hagberg K, Tranberg R. Comparison of plantar pressure in three types of insole given to patients with diabetes at risk of developing foot ulcers - a two-year, randomized trial. J Clin Transl Endocrinol. 2014;1:121.

54. Telfer S, Woodburn J, Collier A, Cavanagh P. Virtually optimized insoles for offloading the diabetic foot: a randomized crossover study. J Biomech. 2017;60:157

55. Tsung BYS, Zhang M, Mak AFT, Wong MWN. Effectiveness of insoles on plantar pressure redistribution. J Rehabil Res Dev 2004;41(6A):767-74.

56. Busch K, Chantelau E. Effectiveness of a new brand of stock 'diabetic'shoes to protect against diabetic foot ulcer relapse. A prospective cohort study. Diabet Med. 2003;20:665.
57. Bus SA, Waaijman R, Arts M, De Haart M, Busch-Westbroek T, Van Baal J, Nollet F. Effect of custom-made footwear on foot ulcer recurrence in diabetes: a multicenter randomized controlled trial. Diabetes Care. 2013;36:4109.

58. Chantelau E, Kushner T, Spraul M. How effective is cushioned therapeutic footwear in protecting diabetic feet? A clinical study. Diabet Med. 1990;7:355.

59. Lavery LA, LaFontaine J, Higgins KR, Lanctot DR, Constantinides G. Shearreducing insoles to prevent foot ulceration in high-risk diabetic patients. Adv Skin Wound Care. 2012;25:519.

60. López-Moral M, Lázaro-Martínez JL, García-Morales E, García-Álvarez Y, Álvaro-Afonso FJ, Molines-Barroso RJ. Clinical efficacy of therapeutic footwear with a rigid rocker sole in the prevention of recurrence in patients with diabetes mellitus and diabetic polineuropathy: a randomized clinical trial. PLoS One. 2019;14:e0219537.

61. Rizzo L, Tedeschi A, Fallani E, Coppelli A, Vallini V, lacopi E, Piaggesi A. Custom-made orthesis and shoes in a structured follow-up program reduces the incidence of neuropathic ulcers in high-risk diabetic foot patients. Int J Low Extrem Wounds. 2012;11:59.

62. Ulbrecht JS, Hurley T, Mauger DT, Cavanagh PR. Prevention of recurrent foot ulcers with plantar pressure-based in-shoe orthoses: the CareFUL prevention multicenter randomized controlled trial. Diabetes Care. 2014;37:1982.

63. https://www.un.org/en/development/desa/policy/wesp/wesp_current/2014 wesp_country_classification.pdf. Country classification. Accessed 5 Dec 2019.

64. Lin T-L, Sheen H-M, Chung C-T, Yang S-W, Lin S-Y, Luo H-J, Chen C-Y, Chan $\mathrm{I}-\mathrm{C}$, Shih $\mathrm{H}-\mathrm{S}$, Sheu $\mathrm{WH}-\mathrm{H}$. The effect of removing plugs and adding arch support to foam based insoles on plantar pressures in people with diabetic peripheral neuropathy. J Foot Ankle Res. 2013;6:29.

65. Patil K, Babu M, Oommen P, Malaviya G. On line system of measurement and analysis of standing and walking foot pressures in normals and patients with neuropathic feet. Innovation et Technologie en Biologie et Médecine. 1996;17:401.

66. Patil K, Bhat M, Bhatia M, Narayanamurthy V, Parivalavan R. New on-line methods for analysis of walking foot pressures in diabetic neuropathy. Frontiers Med Biol Eng. 1999;9:49.

67. Patil K, Srinath M. New image-processing system for analysis, display and measurement of static and dynamic foot pressures. Med Biol Eng Comput. 1990;28:416.

68. Dahmen R, Haspels R, Koomen B, Hoeksma AF. Therapeutic footwear for the neuropathic foot: an algorithm. Diabetes Care. 2001;24:705.

69. Lavery LA, Lanctot DR, Constantinides G, Zamorano RG, Athanasiou KA, Agrawal CM. Wear and biomechanical characteristics of a novel shearreducing insole with implications for high-risk persons with diabetes. Diabetes Technol Ther. 2005;7:638.

70. Bus S, Deursen R, Armstrong D, Lewis J, Caravaggi C, Cavanagh P. Footwear and offloading interventions to prevent and heal foot ulcers and reduce plantar pressure in patients with diabetes: a systematic review. Diabetes Metab Res Rev. 2016:32:99.

71. Healy A, Naemi R, Chockalingam N. The effectiveness of footwear as an intervention to prevent or to reduce biomechanical risk factors associated with diabetic foot ulceration: a systematic review. J Diabetes Complicat. 2013:27:391.

72. Andrews J, Guyatt G, Oxman AD, Alderson P, Dahm P, Falck-Ytter $Y$, Nasser M, Meerpohl J, Post PN, Kunz R. GRADE guidelines: 14. Going from evidence to recommendations: the significance and presentation of recommendations. J Clin Epidemiol. 2013;66:719.

73. Lazzarini PA, Crews RT, van Netten JJ, Bus SA, Fernando ME, Chadwick PJ, Najafi B. Measuring plantar tissue stress in people with diabetic peripheral neuropathy: a critical concept in diabetic foot management. J Diabetes Sci Technol. 2019;13:869.

74. Lillie EO, Patay B, Diamant J, Issell B, Topol EJ, Schork NJ. The n-of-1 clinical trial: the ultimate strategy for individualizing medicine? Personalized Med. 2011;8:161.

75. Tate RL, Perdices M, Rosenkoetter U, Wakim D, Godbee K, Togher L, McDonald $S$. Revision of a method quality rating scale for single-case experimental designs and n-of-1 trials: the 15-item risk of Bias in N-of-1 trials (RoBiNT) scale. Neuropsychol Rehabil. 2013;23:619.

76. Shaw JE, Sicree RA, Zimmet PZ. Global estimates of the prevalence of diabetes for 2010 and 2030. Diabetes Res Clin Pract. 2010:87:4.

\section{Publisher's Note}

Springer Nature remains neutral with regard to jurisdictional claims in published maps and institutional affiliations. 